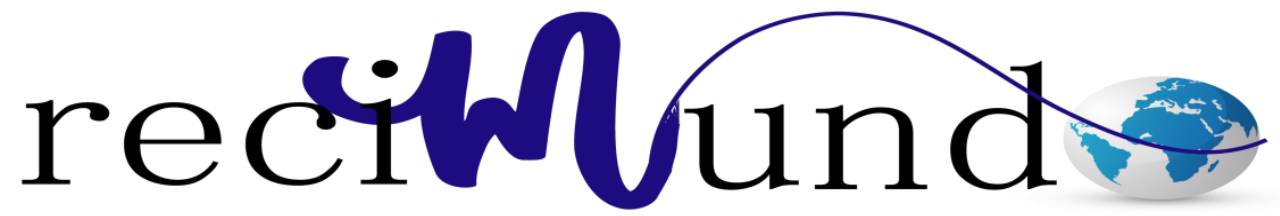

Revista Científica Mundo de la Investigación y el Conocimiento

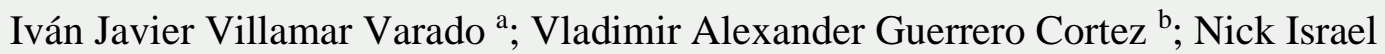
Balseca Villavicencio $^{\mathrm{c}}$

Fomento empresarial del cantón Jipijapa, provincia de Manabí, Ecuador

Revista Científica Mundo de la Investigación y el Conocimiento. Vol. 2 núm.2, mayo, ISSN: 2588-073X, 2018, pp. 3-23

DOI: $10.26820 /$ recimundo/2.(2).2018.3-23

Editorial Saberes del Conocimiento

Recibido: 05/12/2017

Aceptado: 15/03/2018

a. Universidad de Guayaquil; mba.ivanvillamar@gmail.com

b. Universidad de Guayaquil; vladimir.guerrerov@ug.edu.ec

c. Universidad de Guayaquil; nick.balsecav@ug.edu.ec 


\section{Fomento empresarial del cantón Jipijapa, provincia de Manabí, Ecuador}

Vol. 2, núm. 2., (2018)

Iván Javier Villamar Varado;Vladimir Alexander Guerrero Cortez; Nick Israel Balseca Villavicencio

\section{RESUMEN}

Este trabajo investigativo tiene como objetivo describir brevemente los aspectos más relevantes del inicio del desarrollo económico del Cantón jipijapense y cómo es en la contemporaneidad, con la finalidad de explicar la situación particular por la que atraviesa recientemente dicha economía local y así mismo, a manera de conclusión, exponer un esbozo de las actividades económicas que se vienen desarrollando en la actualidad como tendencia, con las que se estima se fomente a corto plazo lograr la recuperación y el fortalecimiento económico en dicha región; para ello la metodología empleada en la presente investigación fue con un enfoque documental, haciendo uso de una revisión bibliográfica a nivel exploratorio-descriptivo, mediante una laboriosa búsqueda tanto en publicaciones hechas en material físico como electrónicos, tales como libros, revistas especializadas, foros, simposios, páginas web, e-book y demás fuentes de información, nacionales e internacionales, legales, oficiales o extraoficiales, respaldadas por profesionales en la materia, en idioma castellano, y en todo momento relacionadas con el tema en cuestión, todo ello con la primordial intención de que la información obtenida sirva para proporcionar conocimiento fundamental al interesado y en particular para esta investigación, sirva de fundamento en de valiosas conclusiones que se desarrollarán a continuación, entre las cuales se destaca que un modelo adaptado ayudará a concretar un liderazgo territorial donde la comunidad de nuevos profesionales, la representación del gobierno local y los demás actores territoriales propicien una el florecimiento económico de la región. De la misma forma se detecta que el tema desarrollo local abarca muchos campos y está siendo introducido con énfasis en los

programas que presenta el gobierno central, cada uno de ellos orientado con el plan nacional que se ha venido desarrollando.

Palabras clave: Desarrollo, economía, actividades, fomentar, fortalecimiento. 


\title{
Fomento empresarial del cantón Jipijapa, provincia de Manabí, Ecuador
}

Vol. 2, núm. 2., (2018)

Iván Javier Villamar Varado;Vladimir Alexander Guerrero Cortez; Nick Israel Balseca

Villavicencio

\begin{abstract}
This research work aims to briefly describe the most important aspects of the beginning of the economic development of the Kipijapense Canton and how it is in the contemporaneity, with the purpose of entering to explain the particular situation that the local economy has recently experienced and likewise, in a way in conclusion, to present an outline of the economic activities that are currently being developed as a trend, with which it is estimated that in the short term it will be possible to achieve recovery and economic strengthening in said region; for this the methodology used in the present investigation was with a documentary approach, making use of a bibliographical revision at exploratory-descriptive level, through a laborious search both in publications made in physical and electronic material, such as books, specialized magazines, forums, symposia, web pages, e-book and other sources of information, national and international, legal, official or unofficial, supported by professionals in the field, in Spanish, and at all times related to the subject in question, all with the The main intention that the information obtained serves to provide fundamental knowledge to the interested party and in particular for this investigation, serves as a basis for valuable conclusions that will be developed below, among which it is emphasized that an adapted model will help to concretize a territorial leadership where the community of new professionals, the represe Local government and other territorial actors encourage the economic flourishing of the region. In the same way it is detected that the topic of local development covers many fields and is being introduced with emphasis on the programs presented by the central government, each of them oriented with the national plan that has been developed.
\end{abstract}

Key words: Development, economy, activities, encourage, strengthening. 


\section{Fomento empresarial del cantón Jipijapa, provincia de Manabí, Ecuador \\ Vol. 2, núm. 2., (2018) \\ Iván Javier Villamar Varado; Vladimir Alexander Guerrero Cortez; Nick Israel Balseca Villavicencio}

\section{Introducción.}

Desde sus inicios, el desarrollo económico de la región de Manabí estuvo enmarcado por su principal actividad, como lo era la producción y exportación de café, además del comercio en sus diferentes formas, la artesanía y posteriormente, el turismo. En específico, el Cantón de Jipijapa está ubicado al sur de Manabí y es conocida también como "La Sultana del Café", por ser el principal productor de café en el Ecuador. (Gobierno Provincial de Manabí, 2016)

Entrando en materia relevante, se puede mencionar que, entre 2006 y 2014, Ecuador experimentó un crecimiento promedio del PIB impulsado por los altos precios del petróleo e importantes flujos de financiamiento externo al sector público. Esto permitió un mayor gasto público, incluyendo la expansión del gasto social e inversiones emblemáticas en los sectores de energía y transporte. En ese periodo, la pobreza disminuyó del 37,6\% al 22,5\% y el coeficiente de desigualdad de Gini se redujo de 0,54 a 0,47 debido a que los ingresos de los segmentos más pobres de la población crecieron más rápido que el ingreso promedio. (Banco Mundial, 2017)

En el mismo informe, se deja claro que, hay riesgo en mantener esos logros, debido a dos principales problemas, los bajos precios del petróleo y la apreciación del dólar. Ese contexto se agrava por un terremoto que afectó la zona costera noroccidental del país el 16 en abril de 2016. Entre 2014 y 2016, el desempleo urbano aumentó de 4,5\% a 6,5\% y el subempleo urbano aumentó de $11,7 \%$ a $18,8 \%$. En este mismo periodo, la pobreza y el coeficiente de Gini de desigualdad permanecieron básicamente estancados.

Agregan que, en ausencia de una moneda local y ahorros fiscales, el gobierno se ha visto en la necesidad de reducir la aún alta inversión pública y contener el aumento del gasto corriente. 


\section{Fomento empresarial del cantón Jipijapa, provincia de Manabí, Ecuador}

Vol. 2, núm. 2., (2018)

Iván Javier Villamar Varado; Vladimir Alexander Guerrero Cortez; Nick Israel Balseca

Villavicencio

Las autoridades también han movilizado diferentes fuentes de financiamiento externo y doméstico y se han realizado algunas reducciones del gasto. Así mismo, se han aplicado medidas temporales para aumentar los ingresos públicos no petroleros y restringir las importaciones. Estas políticas han permitido moderar temporalmente los efectos de los bajos precios el petróleo y financiar la reconstrucción posterior al terremoto, pero también han aumentado los pasivos públicos.

En este periodo complejo, Ecuador enfrenta el desafío de alcanzar los consensos políticos necesarios para adecuar su economía al nuevo contexto internacional, retomar una senda del crecimiento sostenible con mayor participación del sector privado y proteger los avances sociales logrados durante la bonanza. Será crítico mejorar la efectividad y progresividad de la política fiscal con el fin de lograr una consolidación que garantice la estabilidad macroeconómica a tiempo que se protege a la población más vulnerable y preserva la confianza del sector privado. En un contexto en que la inversión pública no podrá continuar siendo motor del crecimiento, es indispensable una mejora sistemática en el clima de inversiones que promueva una mayor participación privada y agilice la movilidad del capital y del trabajo a actividades emergentes. Un sector privado más robusto y flexible podría ayudar a diversificar la economía ecuatoriana, aumentar su productividad y generar empleos de calidad con el fin de promover el desarrollo y continuar con la reducción de la pobreza.

En un informe emitido por Centro de Estudios Latinoamericanos (CESLA, 2018) en el mes de enero pasado, respecto al diagnóstico económico de Ecuador, describen que: a) se observa un estancamiento de la economía tras finalizar 2017, porque la inyección de recursos durante la campaña electoral, financiados principalmente con deuda externa, permitieron 


\section{Fomento empresarial del cantón Jipijapa, provincia de Manabí, Ecuador}

Vol. 2, núm. 2., (2018)

Iván Javier Villamar Varado; Vladimir Alexander Guerrero Cortez; Nick Israel Balseca Villavicencio

incrementar el consumo privado. Pero aun, se redujo la inversión, principalmente la extranjera directa, en un contexto de incremento de la deuda pública. Sin embargo, disminuyó la tasa de desempleo, mientras que las condiciones laborales empeoran debido al incremento del subempleo. Se han reducido las exportaciones de petróleo, lo que refleja un empeoramiento en la producción del sector y la necesidad de aplicar políticas encaminadas a la diversificación; y, b) que Ecuador se encuentra entre los países de América Latina con mayor déficit fiscal, esto es porque, pese al incremento en los ingresos tributarios y a la leve recuperación en los precios del petróleo, crece el endeudamiento. Por este motivo, la política de recortes planeada por el nuevo ejecutivo resulta insuficiente. Si bien ha habido una reducción en los gastos de inversión en obra pública durante el año 2017, otros gastos siguen siendo elevados, como son los gastos de personal. Adicionalmente, es importante agregar la enorme dependencia de la deuda pública externa.

Independientemente de todo explicado, hay que destacar que las pequeñas y medianas empresas (PYME) en la provincia de Manabí son un importante motor de la economía regional, por su contribución al desarrollo económico y al bienestar social de la población, dado que estas empresas generan el mayor crecimiento de la producción regional de servicios y aportan al progreso nacional, siendo agentes económicos que fomentan un importante porcentaje de empleos en cada cantón, y cubren una parte significativa de la demanda de productos y servicios en la región. En relación a esto, el impulso para el desarrollo económico que se promueve por vía Pública, por citar un ejemplo, el llevado adelante el Gobierno Provincial de Manabí en coordinación con el gobierno parroquial y la Fundación Educativa Monseñor Rada (FUNDER), es a través del dictado de talleres de emprendimiento, difusión de la campaña turística “iVen A 


\section{Fomento empresarial del cantón Jipijapa, provincia de Manabí, Ecuador}

Vol. 2, núm. 2., (2018)

Iván Javier Villamar Varado;Vladimir Alexander Guerrero Cortez; Nick Israel Balseca

Villavicencio

Manabí! ¡Explóralo!”, por medio de videos propagandísticos proyectados en canales audio visuales, y en especial por el programa de televisión "Buenas Noticias", entre otras.

Recientemente, en el mes de diciembre pasado, el prefecto de Manabí, durante el desarrollo de las mesas de competitividad convocada por el Ministerio de Industrias y Productividad, que se realizó en la Pontificia Universidad Católica de Manabí, sede Portoviejo, aseguro que durante el año 2017, el Gobierno Provincial de Manabí, a través de la dirección de Fomento Productivo, ha invertido más de 1 millón de dólares en 15 proyectos elaborados por la Unidad de Proyectos de Emprendimientos y Gestión Empresarial, beneficiando a más de 3.600 familias y vinculando a 94 asociaciones de los 22 cantones de la provincia. Además, aseguró que también se ha dado apoyo y acompañamiento técnico a cerca de 500 emprendedores en la provincia y muchos ya están comercializando en las cadenas de supermercados del país. (Zambrano, 2017)

\section{Materiales y métodos}

En vista de que lo que se pretende con este trabajo es explorar el tema de la situación económica coyuntural (a nivel empresarial) de la región Manabí, y en específico en el Cantón Jipijapense, con la finalidad de describir los aspectos generales más resaltantes, y con estos se pueda explicar trascendencia socioeconómica de la región de manera sencilla, y subsiguientemente, a través del análisis de la información recabada se pueda llegar a explicar la situación actual y sus posibles proyecciones, para el desarrollo de la presente investigación se adoptar la de tipo documental, exploratoria y longitudinal. 


\section{Fomento empresarial del cantón Jipijapa, provincia de Manabí, Ecuador}

Vol. 2, núm. 2., (2018)

Iván Javier Villamar Varado;Vladimir Alexander Guerrero Cortez; Nick Israel Balseca Villavicencio

Respecto a la investigación Documental, (Baena, 1985) citado en Ávila (2006), la define como "una técnica que consiste en la selección y recopilación de información por medio de la lectura y crítica de documentos y materiales bibliográficos, de bibliotecas, hemerotecas, centros de documentación e información” (p. 72).

Franklin (1997), igualmente citado en Ávila (2006), respecto a la investigación documental aplicada a la organización de empresas como una técnica de investigación, dice que "se deben seleccionar y analizar aquellos escritos que contienen datos de interés relacionados con el estudio" (p. 13).

Por otra parte, en relación a los Estudios Exploratorios, Ávila (2006) refiere a (Kerlinger, 1983) quien dice que los estudios exploratorios buscan hechos sin el objetivo de predecir las relaciones existentes entre las variables. Se utilizan en situaciones en las que prácticamente no se dispone de información o el PON casi no se ha investigado. En este tipo de situaciones se inicia con un estudio exploratorio con el propósito de "preparar el terreno," Dankhe (1986) igualmente citado en (Ávila, 2006), es decir, se desarrollan a fin de ir documentando el tema de investigación.

Según Ávila (2006) en los Estudios Longitudinales la unidad de análisis es observada en varios puntos en el tiempo.

\section{Resultados.}

Por naturaleza, los gobiernos locales concentran su capacidad de gestión directa y autónoma de los recursos para mejorar o desarrollar nuevos servicios o políticas públicas en los 


\section{Fomento empresarial del cantón Jipijapa, provincia de Manabí, Ecuador}

Vol. 2, núm. 2., (2018)

Iván Javier Villamar Varado;Vladimir Alexander Guerrero Cortez; Nick Israel Balseca

Villavicencio

sectores vivienda, salud, educación, ambiente y turismo, entre otros, a escala cantonal y provincial. En vista de ello, a continuación se mencionan diversos planes y proyectos abanderados desde el gobierno central, y ejecutados a nivel local, a fines de ilustrar que viene haciendo la administración pública para que concurran en articulación actores territoriales con la comunidad en general y la empresa privada, en procura de generar desarrollo local en el Cantón Jipijapa, con el objetivo principal de promover y apoyar soluciones creativas a los problemas y las necesidades de los ciudadanos Jipijapense.

Antes de entrar a las definiciones básicas para comprender un poco más este tema, es necesario destacar lo enunciado por Calle et. al. (2017) cuando asegura que el problema del desarrollo no es un problema de competitividad y de costo relativo del trabajo, sino un problema de activación y valorización de los recursos no utilizados o mal utilizados.

Según Garófoli (1996) se trata de "crear una cultura de la producción, de contribuir a la formación de un saber-hacer, de iniciar el proceso de interdependencia entre los actores locales, de difundir los conocimientos y, paralelamente, de estimular la rivalidad y la competencia al mismo tiempo que la solidaridad y la cooperación"

Ahora, analizando la información obtenida de este trabajo investigativo se puede comprender que la innovación de un emprendedor es el enfoque más prometedor para asegurar un trabajo por cuenta propia en los mercados de trabajo bastante competitivos que prevalecen en los países en desarrollo. La innovación permite a los empresarios crear nuevos productos y servicios que satisfagan las necesidades y expectativas cambiantes de los consumidores en el 


\section{Fomento empresarial del cantón Jipijapa, provincia de Manabí, Ecuador}

Vol. 2, núm. 2., (2018)

Iván Javier Villamar Varado;Vladimir Alexander Guerrero Cortez; Nick Israel Balseca Villavicencio

mercado global. Una comprensión de la innovación es fundamental para explorar cómo el capital humano afecta los esfuerzos innovadores de los empresarios.

Chowdhury (2007) define a la innovación como el proceso mediante el cual los empresarios descubren una idea, la desarrollan y la utilizan para crear nuevos productos, servicios y procesos con fines comerciales.

Ser innovador implica ser creativo y usar efectivamente una idea creativa para mejorar el bienestar de uno mismo y satisfacer a los clientes, así como revisar los productos y procesos existentes con el objetivo de producir una mejor o muy diferente.

Por el contrario, Finnerty (2003) citado en (Torrech et. al., 2017) argumenta que un emprendedor es un individuo que coordina diversos recursos para ejecutar una idea innovadora. El emprendimiento innovador, por lo tanto, implica la organización de otros factores de producción para implementar una nueva idea, producto o servicio. La capacidad de los empresarios, especialmente en los países en desarrollo, para desempeñar eficazmente este papel innovador depende en gran medida del capital humano.

Es imperativo observar que la definición de innovación empresarial sería incompleta sin el concepto de capacidades de innovación. Según Gorodnichenko y Monika (2013) también referido en (Torrech et. al., 2017) afirman que las capacidades de innovación abarcan conocimientos, habilidades y enfoques de gestión que apuntan a la modificación y desarrollo del producto. En el contexto de los países en desarrollo, el espíritu emprendedor es una función de la capacidad individual para generar información e ideas que crean valor agregado a los emprendimientos empresariales. 


\section{Fomento empresarial del cantón Jipijapa, provincia de Manabí, Ecuador}

Vol. 2, núm. 2., (2018)

Iván Javier Villamar Varado;Vladimir Alexander Guerrero Cortez; Nick Israel Balseca

Villavicencio

La demanda de un posible desarrollo desde lo local, desde abajo, desde la base o endógeno remite, pues, al caso particular en que la transformación del sistema económico descansa en un tipo de organización política, social y económica que pueda garantizar un margen de autonomía en la definición de las estrategias necesarias, basándose en la utilización de los recursos locales y en las capacidades de controlar los procesos de acumulación y de innovación, de reaccionar a las presiones exteriores y de introducir las formas específicas de regulación social que favorezcan los elementos precedentes eso es lo que expresan Courlet y Pecqueur, B. (1996) en (Torrech et. al, 2017).

Un referente por excelencia está dado por los denominados "sistemas productivos localizados" de alto dinamismo económico basado en la innovación y cuya organización evidencia la importancia de las relaciones colaborativas entre las empresas y de éstas con las instituciones locales que intervienen para apoyar su desarrollo, traduciendo así la articulación de los subsistemas productivo y socio-institucional, que en el caso de la provincia de Manabí no se logra desarrollar esa articulación y dinamismo debido a que no hay quien lidere dicho proceso, lo cual no mejora la calidad de vida de los habitantes muy a pesar de tener los recursos naturales y humanos necesarios para desarrollarse.

En lo que respecta a la cooperación internacional se debe tomar en cuenta que existen recursos internacionales que pueden contribuir al desarrollo local y por lo tanto se debe captarlos, pero hay que destacar que debido al proceso aún centralizado en el Ecuador los Municipios u otros entes públicos no pueden acceder a un crédito internacional si no cuentan con el aval o con la garantía del Estado, esto ocasiona una limitante mayor cuando de por medio existen diferencias políticas con determinados alcaldes y las autoridades de Gobierno, por lo 


\section{Fomento empresarial del cantón Jipijapa, provincia de Manabí, Ecuador}

Vol. 2, núm. 2., (2018)

Iván Javier Villamar Varado; Vladimir Alexander Guerrero Cortez; Nick Israel Balseca Villavicencio

tanto los municipios que en algunos casos si reciben recursos internacionales lo hacen únicamente como donaciones o ayudas a determinados programas sociales, lo cual no necesariamente genera sostenibilidad y desarrollo.

En el informe "Ecuador: Una economía en evolución” por (Kreab \& Gavin Anderson Worwide, s.f.) se explica que en Ecuador, a pesar del crecimiento vigoroso de su economía durante los últimos años, sigue dependiendo ampliamente de la inversión pública y las exportaciones de materias primas y en particular del petróleo. Desde el primer cuarto del siglo XX, comenzó su explotación en la Península de Santa Elena, y décadas más tarde, se inició en la región amazónica, convirtiéndose en uno de los principales exportadores de este recurso. Pero por si fuese poco, las riquezas naturales ecuatorianas son vastas, calculándose que en el Golfo de Guayaquil hay reservas de gas de unos 300 mil millones de pies cúbicos. Otros productos de importancia para la economía del país han sido y lo son, el banano, el café, el cacao, el camarón, la madera y el atún, y, en los últimos años, las flores. Existen también productos no tradicionales de exportación, como ciertas especies de frutas y verduras que poco a poco están ganando terreno en el mercado internacional. Esta estructura económica, se complementa con grandes industrias de la maderera y textil, también hay pequeñas empresas de artesanía de gran riqueza por su variedad, sobre todo en campo textil. Por su parte, el turismo está en el cuarto lugar en cuanto a la generación de divisas.

Ecuador, donde el petróleo constituye la principal fuente de generación de divisas y riqueza del país, también constituye el principal riesgo por la variación su precio. Para superar esta situación, el Gobierno se encuentra inmerso en transformar una economía básicamente extractiva y petrolera, hacia una economía más diversificada, incorporado el conocimiento y la 


\section{Fomento empresarial del cantón Jipijapa, provincia de Manabí, Ecuador}

Vol. 2, núm. 2., (2018)

Iván Javier Villamar Varado;Vladimir Alexander Guerrero Cortez; Nick Israel Balseca

Villavicencio

innovación a su sistema productivo, para lo cual se encuentra potenciando la educación a la vez está elevando el nivel académico con Ley de Educación Superior. Paralelamente construye la ciudad del conocimiento y la innovación "Yachay" que representa un foco avanzado y puntero para la innovación, crecimiento económico y la modernización del país con la idea de poder ser un referente para América Latina.

El entorno económico, social, cultural y político es crítico para el surgimiento de nuevas empresas, como para su evolución y crecimiento en particular, la fortaleza en este factor es que haya una formación profesional y educación continua tanto como para los que están a una edad de estudiar y para los que no pueden darles capacitaciones y que puedan entender la innovación del emprendimiento. Los emprendedores en general no disponen de suficiente capital propio, y el soporte financiero está caracterizado por inversión informal tales como: familiares, amigos, terceros privados, etc. (Caicedo, 2016) citado en (Méndez et. al., 2017)

Méndez et. al. (2017)refiriendo a (Viniegra, 2016) define que el Bien Común es una iniciativa social para el servicio de los ciudadanos que tengan valores y poder alcanzar una sociedad sostenible y más equilibrada. Cuando el conocimiento de sí mismo y del contexto se profundiza a través de la crítica, la determinación de luchar por la dignidad propia y ajena es un resultado de la búsqueda que se extiende desde el sí mismo a la familia y allegados, y en la medida de su vigor se proyecta a otras colectividades con las que se comparten intereses y aspiraciones.

Seguidamente, refieren a (Coraggio, 2015) para decir que en la Economía del Bien Común es necesario proporcionar mejora en las empresas para un buen desarrollo de producto o 


\section{Fomento empresarial del cantón Jipijapa, provincia de Manabí, Ecuador}

Vol. 2, núm. 2., (2018)

Iván Javier Villamar Varado;Vladimir Alexander Guerrero Cortez; Nick Israel Balseca Villavicencio

servicio y de esa manera equilibrar en la productividad económica y generar ingresos al país sobre la base de un sistema social, político y económico para un beneficio global, de una manera de dignidad humana, equidad, solidaridad y sostenibilidad ecológica para una buena justicia social y edificar el cambio para alcanzar el objetivo.

Relación entre Economía del Bien Común y la Acción de Emprender

La sociedad hoy en día busca que las organizaciones o empresas satisfagan sus necesidades, y que genere economía productiva al país y que además tenga que ser amigable con el medio ambiente y que impulse de alguna manera el desarrollo de la sociedad, a todo esto, se conoce como responsabilidad social. De esta manera los emprendedores, individuos o grupos deben de reformular la manera de hacer negocio, la concepción del trabajo, el objetivo de las empresas, armonía con el medio ambiente y recuperar valores y conductas que se han perdido

La responsabilidad social, sus valores y su política de sostenibilidad de tal manera que estos se conviertan en los principios orientadores del que hacer de la empresa y que las personas puedan encontrar oportunidades con el tiempo de que ellos vayan planificando sus empresas.

Introducción de la Economía del Bien Común hacia la Acción de Emprender

García (2016) citado en Méndez et. al.(2017) sugiere que el desarrollo de estos planteamientos se requiere de empeño y responsabilidad, esto va a permitir ayudar a cada uno de los que quieran emprender y los que tienen poco conocimiento para emprender un negocio que conlleve la responsabilidad social para ellos nos planteamos objetivos de tal manera que se vayan a cumplir: 


\section{Fomento empresarial del cantón Jipijapa, provincia de Manabí, Ecuador}

Vol. 2, núm. 2., (2018)

Iván Javier Villamar Varado;Vladimir Alexander Guerrero Cortez; Nick Israel Balseca

Villavicencio

o Poder difundir los principios de la Economía del Bien Común.

o Presentar la Economía del Bien común como una herramienta social.

o Elaborar capacitaciones sobre la manera de Emprender con la Economía del Bien Común.

o Elaboraciones de proyectos de Responsabilidad Social.

A todo esto, va a servir para poder saber cómo implementar una empresa de una buena manera introduciendo lo son los valores, a que creen un producto o servicio de la manera correcta, que no dejen de lado lo que es el medio ambiente, la Responsabilidad social, etc.

\section{Crecimiento económico y actividad emprendedora}

Parece existir un amplio consenso con respecto a que la clave para conseguir crecimiento económico y mejoras en la productividad se encuentra en la capacidad empresarial de una economía y en general, que la creación de empresas representa el motor de las economías en los países desarrollados (Audretsch, 2014). Los resultados obtenidos no tienen un carácter uniforme, sino que varían en función del ámbito geográfico, las variables y los indicadores utilizados, así como si el carácter del emprendimiento es por necesidad o por oportunidad.

Con la idea de que el espíritu empresarial es fundamental para el crecimiento económico y con el desarrollo ha llegado una serie de iniciativas de iniciativa empresarial para aumentar la actividad empresarial Minniti (2017) citado en (Audretsch, 2014) sugiere que la política de espíritu empresarial puede ser productiva o improductiva. En el mundo desarrollado, una política 


\section{Fomento empresarial del cantón Jipijapa, provincia de Manabí, Ecuador}

Vol. 2, núm. 2., (2018)

Iván Javier Villamar Varado;Vladimir Alexander Guerrero Cortez; Nick Israel Balseca Villavicencio

común utilizada para promover el emprendimiento (aparentemente productivo) es la introducción de capital de riesgo público que se proporciona a las nuevas y pequeñas empresas y se financia a través de las finanzas públicas. (Audretsch, 2014)

A manera de cierre de esta sección es imprescindible mencionar el "Plan Nacional de Desarrollo 2017-2021 Toda una Vida" de Ecuador, puesto que es el principal instrumento del Sistema Nacional Descentralizado de Planificación Participativa (SNDPP. Este se fundamenta en los logros de los "últimos 10 años" y pone en evidencia la existencia de nuevos retos por alcanzar, en torno a tres ejes principales: 1) Derechos para todos durante toda la vida; 2) Economía al servicio de la sociedad; 3) Más sociedad, mejor Estado, que contienen a su vez tres objetivos nacionales de desarrollo que rompen con la lógica sectorial y dan cuenta de las prioridades que tiene el país. Esta visión se enmarca, también, en los compromisos internacionales de desarrollo global, como la Agenda 2030 y sus Objetivos de Desarrollo Sostenible. (Secretaría Nacional de Planificación y Desarrollo (SENPLADES) de Ecuador, 2018)

En lo que respecta al tema, es importante destacar que se definen tres objetivos dentro del Eje 2 de dicho plan, que son:

- Consolidar la sostenibilidad del sistema económico social y solidario, y afianzar la dolarización.

- Impulsar la productividad y competitividad para el crecimiento económico sustentable de manera redistributiva y solidaria.

- Desarrollar las capacidades productivas y del entorno para lograr la soberanía alimentaria y el desarrollo rural integral. 


\section{Fomento empresarial del cantón Jipijapa, provincia de Manabí, Ecuador}

Vol. 2, núm. 2., (2018)

Iván Javier Villamar Varado; Vladimir Alexander Guerrero Cortez; Nick Israel Balseca

Villavicencio

Demostraciones de éstos objetivos se manifiestan en la acción del estado, a través de su representantes, en todas las negociaciones y acuerdos que sigue celebrando el país con socios comerciales tales como, Alemania,China, España, Italia, entre otros.

\section{Conclusiones.}

Calle, et. al. (2017) refiere que, a nivel internacional, se ha iniciado hace más de treinta años un importante giro en las políticas de desarrollo, que hasta entonces se imponían "de manera descendente" mediante políticas macroeconómicas globales alejadas de las realidades locales y que desde entonces pasan a ser tratadas "de manera ascendente" mediante políticas mixtas, macro y microeconómicas, centradas en promover el protagonismo local.

Continua diciendo que, excepcionalmente, quien marca una inflexión definitiva de esta tendencia a nivel mundial es la Organización de las Naciones Unidas (ONU) con la puesta en marcha del Programa de las Naciones Unidas para el Desarrollo (PNUD), quienes estableciendo dos principios esenciales: $1^{\circ}$ ) Pensar en global y $2^{\circ}$ ) actuar localmente, es decir, adaptar las políticas genéricas (macro) a los casos concretos (micro) de cada entorno local, es el desafío para que exista la participación de las comunidades locales en sus planes de desarrollo, lo que se interpreta como, convertir a las administraciones locales en los principales impulsores del desarrollo como fórmula para ajustarse al máximo a las necesidades y peculiaridades del entorno local. Este nuevo modelo de ayuda al desarrollo no está centrado únicamente en el progreso económico, sino también en el progreso humano sostenible y sustentable, siendo una de sus políticas principales el fomento de la cooperación entre los distintos agentes de una localidad 


\section{Fomento empresarial del cantón Jipijapa, provincia de Manabí, Ecuador}

Vol. 2, núm. 2., (2018)

Iván Javier Villamar Varado;Vladimir Alexander Guerrero Cortez; Nick Israel Balseca Villavicencio

(individuos, administración pública, organizaciones no gubernamentales, empresas, universidades, cooperación internacional, etcétera). (Resumen Latinoamericano, 2013)

En América Latina y el Caribe existe un amplio universo de iniciativas locales que constituyen una muy valiosa materia prima para la acumulación de conocimiento acerca del cómo generar desarrollo local. Con éste propósito en el Ecuador organismos como la Secretaria Nacional de Planificación y Desarrollo (SENPLADES) lidera el proceso de planificación nacional mediante la articulación de las instituciones del sector púbico incluyendo a los Gobiernos Autónomos Provinciales, Cantonales y Parroquiales, y lo hace con un instrumento de planificación denominado Plan de Ordenamiento y Desarrollo Territorial (PDOT) donde se encuentra plasmado el diagnóstico del territorio y los proyectos que requiere la población.

Siguiendo a lo publicado en Resumen Latinoamericano (2013), se puede asegurar que, en definitiva, si bien es cierto que la economía ecuatoriana se ha visto fuertemente afectada por la caída del precio de las materias primas y por la apreciación del dólar, no es menos cierto que, buena parte de las medidas económicas del Gobierno Nacional han consiguió estabilizar la economía. Así mismo, es importante destacar que aunque la inversión pública se redujo debido a la falta de liquidez, esta no se hizo de forma drástica, lo que permitió que se mantuvieran las inversiones ya iniciadas que en muchos casos, en muy corto plazo, comenzaron a revertir utilidades para las finanzas públicas de ese mismo ejercicio económico, situación que se presentó (por mencionar algunos casos) en los proyectos hidroeléctricos, proyectos multipropósito o la refinería de Esmeraldas. 


\section{Fomento empresarial del cantón Jipijapa, provincia de Manabí, Ecuador}

Vol. 2, núm. 2., (2018)

Iván Javier Villamar Varado;Vladimir Alexander Guerrero Cortez; Nick Israel Balseca

Villavicencio

Otro factor determinante con la que se lidió la escasez de recursos derivados de la restricción externa, fue el fortalecimiento del sistema fiscal ecuatoriano, el cual dota al Estado de una mayor capacidad de hacer frente a coyunturas económicas adversas.

Las cifras mostradas por la economía ecuatoriana a partir del segundo trimestre del año pasado, unidas a la estabilización (aún en el corto plazo) de los precios del petróleo con una leve tendencia alcista, coadyuvó en el cierre de ejercicio económico ecuatoriano del 2017, y de cierta manera abonó el terreno para que, en el marco del plan de desarrollo económico adelantado desde la administración del gobierno central, los proyectos de recuperación, promoción, apoyo al emprendimiento y fomento de la economía local en el Cantón Jipijapense siente bases más sólidas que posibiliten aún más su permanencia y crecimiento en el tiempo.

Según los datos aportados por el CESLA (2018), se espera en Ecuador que el crecimiento se modere. La incertidumbre política que conlleva el próximo referéndum que tendrá lugar el 4 de febrero, va a generar inestabilidad en las variables económicas que han contribuido a mantener el crecimiento económico, consumo privado y exportaciones. Por otro lado, el nuevo Gobierno está acometiendo reformas fiscales, junto con un proceso de privatización, que van a contribuir a reducir los niveles de formación bruta de capital fijo. Asimismo, la evolución en los precios del petróleo no resulta suficiente para recuperar las exportaciones. Por último, los esfuerzos por diversificar la economía aún son insuficientes.

La deuda pública va a continuar creciendo, pese a los esfuerzos del nuevo Gobierno por reducir el elevado déficit fiscal. Esta situación plantea un escenario de incertidumbre respecto de la sostenibilidad de la deuda pública. Los recortes acometidos por el Gobierno son insuficientes. 


\section{Fomento empresarial del cantón Jipijapa, provincia de Manabí, Ecuador}

Vol. 2, núm. 2., (2018)

Iván Javier Villamar Varado;Vladimir Alexander Guerrero Cortez; Nick Israel Balseca Villavicencio

Pese al incremento en los ingresos recaudatorios y el proceso de privatización, es necesario recortar el gasto público, principalmente en inversiones de capital, con el objetivo de reducir el déficit fiscal. El precio del petróleo se recupera gradualmente, lo que va a ayudar a recortar dicho déficit en el futuro. Sin embargo, aún es necesario continuar acudiendo a la deuda pública.

\section{Recomendaciones.}

Se cree relevante que en un futuro se investigue en esta misma línea, sobre los resultados obtenidos a mediano plazo post participación conjunta entre gobierno, sociedad y/o empresas privadas, en los proyectos de emprendimiento empresarial, reactivación de la producción nacional y demás planes estratégicos desarrollados en el marco del proyecto nacional de rescate de la economía ecuatoriana.

\section{Bibliografía.}

Ávila, H. (2006). Introducción a la Metodología de la Investigación. eumed.net.

Banco Mundial. (11 de 04 de 2017). Banco Mundial. Obtenido de http://www.bancomundial.org/es/country/ecuador/overview

Calle, A., Calle, J., \& Yoza, N. (2017). Modelo de Articulación Universidad y Actores Territoriales Para El Desarrollo Local de la Provincia de Manabí-Ecuador. DELOS: Desarrollo Local Sostenible, 10(29), 18. Obtenido de www.eumed.net/rev/delos/29

Cazau, P. (2006). Introducción a la investigación en ciencias sociales (Tercera ed.). Buenos Aires: Buenos Aires.

CESLA. (2018). Tendencias Latinoamericanas. Centro de Estudios Latinoamericanos. Madrid : Universidad Autónoma de Madrid. Obtenido de https://www.cesla.com/archivos/Informe_economia_Ecuador_enero_2018.pdf

Chowdhury, N. (2007). An Empirical Analysis of Selected Factors Affecting Corruption in the Asia-Pacific Region. Ritsumeikan Journal of Asia Pacific Studies, 14. Obtenido de http://www.apu.ac.jp/rcaps/uploads/fckeditor/publications/journal/RJAPS_V27_Navil.pd $\mathrm{f}$ 
Fomento empresarial del cantón Jipijapa, provincia de Manabí, Ecuador

Vol. 2, núm. 2., (2018)

Iván Javier Villamar Varado;Vladimir Alexander Guerrero Cortez; Nick Israel Balseca

Villavicencio

Garófoli, G. (1996). Industrialization diffuse et systemes productifs locaux: un modele difficilement transférable aux pays en voie du développement, en Abdelmalki, L. y Courlet, C., Les nouvelles logiques du développement. Paris: L'Harmattan.

Gobierno Provincial de Manabí. (2016). www.manabi.gob.e. Recuperado el 20 de 02 de 2018, de www.manabi.gob.e: http://www.manabi.gob.ec/cantones/jipijapa

Kreab \& Gavin Anderson Worwide. (s.f.). Ecuador: Una economia en evolución. Quito: Kreab Gavin Anderson LatAm. Obtenido de http://contenidos.ceoe.es/resources/image/una_mirada_a_ecuador.pdf

Méndez, Reyes, \& Urrutia. (2017). Economía del Bien Común y Acción de Emprender. CE: Contribuciones a la Economía. Obtenido de http://eumed.net/ce/2017/4/economia-biencomun.html

Resumen Latinoamericano. (2013). Resumen Latinoamericano. Obtenido de Resumen Latinoamericano: http://www.resumenlatinoamericano.org/2016/11/25/informecoyuntura-economica-ecuador/

Torrech, J., Orellana, N., \& Yance, C. (2017). El rol del capital humano en la. Contribuciones a la economía, 25. Obtenido de http://eumed.net/ce/2017/1/innovacion.html

Zambrano, M. (Diciembre de 2017). Se efectuaron mesas de competitividad en manabí. Obtenido de http://www.manabi.gob.ec/11927-se-efectuaron-mesas-competitividadmanabi.html 